\title{
Tolerance Design of Robot Parameters Using Generalized Reduced Gradient Algorithm
}

\author{
Trang Thanh Trung, Li Wei Guang, and Pham Thanh Long
}

\begin{abstract}
In robot design, how to allocate tolerances for parts in manufacturing and assembling of robot is very important because this directly affects product quality and manufacturing cost. This paper introduces a technique using the Generalized Reduced Gradient algorithm optimization to allocate tolerances into robot parts. This method consists of three steps. First, based on the particular structure of robot, various methods are considered before the best method suitable for modeling the associated equation is chosen. Then, a mathematical model for tolerance allocation is formulated and transferred into the non-linear multi-variable optimization problem. Finally, this optimization problem is solved by using the Generalized Reduced Gradient algorithm. Two examples are used to verify the feasibility of the proposed method; the accuracy and effectiveness of the proposed method in producing the tolerance allocations is also illustrated via calculation and simulation results.
\end{abstract}

Index Terms-Industrial robot, tolerances, optimization problem, generalized reduced gradient algorithm.

\section{INTRODUCTION}

Robots are usually employed in industrial and medical applications to position or orient an object, where high accuracy, repeatability and stability of operations are required. The repeatability is a measure of the ability of the robot to move back to exactly the same pose over and over again, while accuracy is defined as the ability of the robot to precisely move to a desired pose in 3-D space [1]. Unacceptable performance, which is the positional and directional deviation of the robot end effector, may be caused by a number of sources such as the joint clearance of actuators and controllers, manufacturing and assembling errors, different types of measurement and control errors, elastic deformations of structural components, and so on [2]-[4]. As a matter of the fact, all these errors are random in nature and especially there is no way of eliminating the dimensional tolerances and clearances prescribed on the manufacturing and assembling operations [5]. Hence the requirement of selecting tolerance within the smallest possible is emerged.

Manuscript received August, 26, 2015; revised January 5, 2016. This work was supported by The Chinese National High-Tech Research and Development Program (863 Program), Project number 2015AA043005.

Trang Thanh Trung and Li Wei Guang are with the School of Mechanical and Automotive Engineering, South China University of Technology, China (e-mail: trangthanhtrung@gmail.com,wguangli@scut.edu.cn).

Pham Thanh Long is with the Faculty of Mechanical Engineering, Thai Nguyen University of Technology, Viet Nam (e-mail kalongkc@gmail.com).
The smaller the tolerance, the better the product quality but the higher the manufacturing cost. It is always not an easy task to choose from a proper balance between the product quality and the manufacturing cost [6]. Traditionally, these parameter tolerances are mostly selected by experience and intuition of designers, handbooks, and standards, which lead to some errors [7]. So the product quality is not guaranteed and the manufacturing cost may be higher than necessary. On the other hand, designing robot to satisfy the desired performance requirement is a complex activity because of the nonlinear and coupled relationship between the robot actuators and end-effectors and uncertainties presenting in kinematic parameters. These uncertainties attribute to error factors in robot systems, which cause variability in performance. Therefore, how deviation of link dimensions and joint tolerances in the robot systems contributing to the end-effector deviation and what analysis method being used to evaluate the performance are the crucial issues.

In this paper, a technique using Generalized Reduced Gradient (GRG) optimization algorithm to solve the tolerance design robot problem is proposed. This proposed method can be applied to both robot arm and parallel robot, and its procedure is described with two processes: direct and inverse. The results of two examples simulation are presented to demonstrate the accuracy and effectiveness of this method.

\section{LITERATURE REVIEW}

As the aforementioned, in robot design selection of optimum design and process parameter tolerance of robot is a challenging task. In order to solve this problem, a number of researches have been developed using techniques as conventional optimization methods, quality engineering methods, genetic algorithms (GA), simulated annealing (SA), neuro-fuzzy learning, and so on and some are already in regular use, statistical planning of experiments, numerical simulation procedures, and probabilistic modeling.

Many researchers consider tolerance allocation as an optimization problem in which the tolerance values of parts are taken as the control variables, and the machining costs are taken as the objective function to be minimized. Michael and Siddall extended the conventional design optimization problem, in which the nominal values of the design variables are of interest, to include the optimal allocation of manufacturing tolerances [8]. Parkinson provided an application in which tolerances of a system is selected using optimization technique [9]. In Weidong Wu, S. S. Rao's research, they focus on the optimal allocation of joint tolerances with consideration of the positional and directional errors of the robot end effector and the manufacturing cost 
[3]. Similar Rao R. S et al. [10], the interval analysis is used for predicting errors in the performance of robot. By using Denavit-Hartenberg $(\mathrm{DH})$ rule for modeling the kinematic problem, the objective functions include the dimensional tolerances (d in DH table) and assembly errors (in DH table). These errors, however, have no relationship with the level of free movement along the centripetal direction of the joints which can lead to the choice of accurate level of bearing. The unknown joint variables are modeled as interval parameters due to the inherent uncertainty. The cost-tolerance model is assumed to be of an exponential form during optimization, in optimum status this value reach the minimum. However, in their work they have not yet mentioned the way of identifying the tolerances of each joint, as well as the application on parallel robot.

The optimization-based tolerance approach methods tend to be impractical as the complexity of the robot assemblies increases. Gadallah and ElMaraghy [11] are one of the first researchers attempting to apply the parameter design techniques of quality engineering to the tolerance optimization problem through the concept of the quality loss function. Many researchers, like Choi H-GR, Park M-H [12], Cho B-R, Kim YJ [13] and Feng C-X, Wang J [14] found in their studies the concept of loss function in the tolerance allocation problem. An extensive review of the methodologies to obtain robust design of products that have low performance variation caused by the variations of control factors and noise factors is given by Rout and Mittal [15].

Another group putting efforts on overcome the impracticality of optimization-based tolerance allocation problems give birth to some novel approaches based on relatively new techniques, such as GA, neural networks, SA and fuzzy logic [16]. Paredis and Khosla proposed a distributed agent-based GA approach to create fault tolerant serial chain manipulators from a small inventory of links and modules [17]. Coello et al. developed new technique that combines GA and the weighted min-max multi-objective optimization method for robot design [18]. Zhuang et al. applied GA to select the optimal robot measurement configurations, which is an important element in robot calibration [19] while Ji S. et al. [20], [21] and Chen T-C and Fischer G. W. [22] applied the GA to the tolerance allocation problem. Later Zhang. D et al. implemented GA to obtain the optimum design of parallel kinematic tool-heads considering the global stiffness and workspace volume [23]. Subsequently a method based on GA was introduced by P.T. Zacharia and N. A. Aspragathos for the determination of the optimal sequence of a non-redundant manipulator end-effector, considering multiple configurations [24]. On the other hand, Kopardekar and Anand [25] applied neural network techniques to tolerance allocation. While Dupinet E. et al. [26] exploited fuzzy logic and SA for the purpose of efficiently dealing with the machine capability and manufacturing specific phenomena, such as mean shift, the back propagation method is used to train the network that generates the part tolerances. The SA was discussed for optimum kinematic design of serial link manipulators [27] and it was adopted to obtain near optimal measurement configurations of robot for calibration [28].

Due to the random nature of link dimensions and joint clearances, Jeong Kim et al. proposed a stochastic approach to figure out the reliability for the open-loop mechanism [5]. In their work, with the assumption that all kinematical parameters are the normally distributed random variables, the stochastic model of the links with dimensional tolerances and of the revolute joints with clearances is presented. The kinematical reliability for the positioning and orientation repeatability is then calculated analytically based on an advanced first-order second moment method. Bhatti and Rao developed a probabilistic approach combined with Monte Carlo simulation method to the manipulator kinematics and dynamics taking into account the relationships among the geometric tolerances, arm configuration and manipulator reliability [29], [30]. Lee and Woo [31] formulated the tolerance synthesis as a probabilistic optimization problem in which a random variable is associated with a dimension and its tolerance. A general probability density function of the endpoints of planar robots based on probability theory was established by Zhu and Ting [32] to find the probability of the robot end point locations with a desired tolerance zone and to determine the joint clearance value. Furthermore, they offered a kinematical model to understand the effect of joint clearances and to determine the directional deviation of single and multiple degree-of-freedom linkages against the worst case [33]. By using probabilistic approach, Rao and Bhatti proposed manipulator reliability to express its kinematic and dynamic performance. Where the manipulator reliability is defined as probability of end effector pose falling within a specified range from the desired pose [34].

An alternative method regularly used is the statistical experiments. Riemer and Edan observed experimentally that there is a statistically significant difference among the repeat abilities at both different locations in the workspace and the different height of the target point [35]. Parametric tolerance design of a manipulator which used full factorial design of experiment (DOE) approach without taking noise factor into consideration had been attempted by B.K. Rout and R.K. Mittal [36]. They had applied the Taguchi method to find the optimal parameters settings for improving the quality of performance of a manipulator [37]. They also has been proposed a hybrid technique which combines the evolutionary optimization technique and orthogonal array of the Taguchi method to optimize the design parameter tolerances such as link dimensions, link inertias, and actuating torque fluctuations. These parameter tolerances actually would deliver specified level of performance measure with minimum manufacturing cost. The hybrid approach proposed is the best choice for the purpose of parameter tolerance design considering the effect of noises for performance simulation [4], [38]. However, this technique is an off-line procedure and among the manufacture errors, it can solve only the tolerances of link dimensions which are the geometrical error while the problem of determining the limited tolerances of the dynamic joints in robot is not considered.

For the optimal design of robot parameter tolerance, some researchers also used the approach based on Jacobian matrix. Maciejewski investigated the pose in which a redundant serial manipulator would have optimum dexterity in case of jam in any of its joints by using the singular values of the Jacobian matrix [39], [40]. Lewis and Maciejewski defined a fault 
tolerance measure for joint jam in the redundant serial manipulators based on the smallest singular value of the Jacobian matrix [41]. Later, Mahir Hassan and Leila Notash applied a method based on the Jacobian matrix to determine the joint tolerance which satisfies both economic and technology objectives. In this work, the type and the potential locations of the redundant backup joints are assumed to be pre-identified and their axes directions are identified by employing the Lagrange multiplier optimization method [42]. This solution therefore can be applied only to the fully actuated robot but the parallel robot where the tolerances of several passive joints cannot be solved by this solution. Moreover, it is complicated to calculate the optimal manufacture tolerance based on Jacobian matrix because of using the Lagrange multiplier method for eliminating the cross-affect among the tolerances. Recently, Phan Bui Khoi et al. has been used an approach based on the differential of Jacobian matrix and GA method to identify the positional and directional errors of the end effector along with the geometrical errors of the intermediate links and joints. The inverse mechanism of the tolerance problem however has not been mentioned in this approach [43].

For the investigation of the relationship between the pre-identified errors in the component links and the positional and directional accuracy in the end effector, Miomir Vukobratovic and Branislav Borovac introduced a map error index method (MEI). By changing links parameters deviations, a whole family of portraits with the accompanying deviations may be obtained [44]. For the purpose of evaluation of the robot kinematic accuracy through the computation of its responses with and without clearance, a coefficient of performance $M E I=\sqrt{\frac{\sum_{1}^{k}\left(\frac{1}{\text { index_value }}\right)^{2}}{k}}$ is drawn as a figure illustrating the robot response in the form of error chart. Based on this information obtained, in the stage of the robot assembly, the selection of the links with appropriate deviations will be performed to ensure a desired accuracy of the robot end effector. It is relatively easy for investigation procedure but the inverse mechanism, the effect of the end-effector deviation on both tolerances of link dimensions and deviations of joints has not been showed.

From the discussions above, it can be concluded that there are many available efficient methods applying to robot issues but the problem of identifying the tolerances of links dimension and joints applying in robot arm and parallel robot with both direct and inverse directions is occasionally considered. Hence, a new approach relating to the determination of tolerances of links dimension and joints satisfying the mentioned objectives is proposed in this paper. In which, the tolerance allocation including the tolerances of the links and joints are represented as an optimization problem and a new mathematical model based on RosenBock Banana function is established and solved by using the Generalized Reduced Gradient algorithm. The tolerance allocation for the robot arm and parallel robot industrial assembly is produced by the above method, and the results show that the method can be used in design with the optimal tolerance values of parts.

\section{THE FORMATION OF THE OPTIMAL PROBLEM}

The associated equations are the fundamental basis to form the kinematic problems of robots. In building associated equations, general principles are based on closed-loop vectors. Depending on each particular structure of robots, the inter-relations of reference systems can be modeled in the form of closed-loop vectors. The diagram of closed-loop vectors in both robot arm and parallel robot is illustrated in Fig. 1.
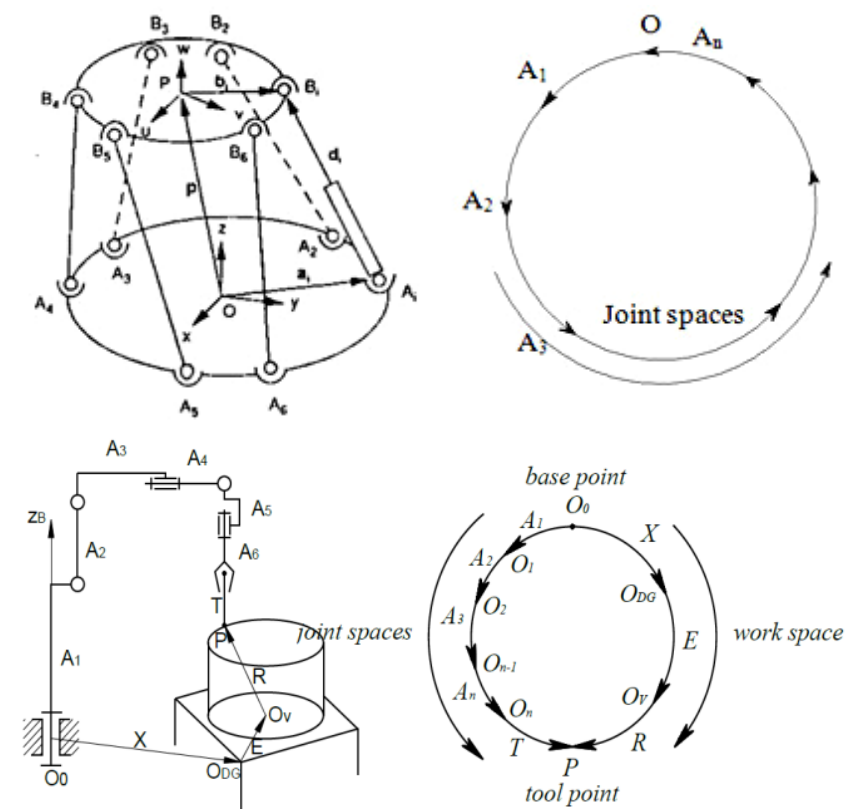

Fig. 1. Diagram of closed-loop vectors in robot arm and parallel robot.

The relationship is written as the loop vector equation as follows:

For parallel robot:

$$
A_{1} A_{2} \ldots A_{n}=I
$$

For robot arm:

$$
A_{1} A_{2} \ldots A_{n}=X . E . R . T^{-1}
$$

The matrices on the left hand side of these equations are determined by the $\mathrm{DH}$ rule, screw transpose rule or geometric rule depending on the selection of the users. The right hand side matrices illustrate the position and direction of the end effector and they are determined based on the control trajectory of robot in space. The expansion the equation $(1,2)$ can be rewritten as:

$$
\left|\begin{array}{cccc}
n_{x} & s_{x} & a_{x} & p_{x} \\
n_{y} & s_{y} & a_{y} & p_{y} \\
n_{z} & s_{z} & a_{z} & p_{z} \\
0 & 0 & 0 & 1
\end{array}\right|=\left|\begin{array}{cccc}
a_{11} & a_{12} & a_{13} & a_{14} \\
a_{21} & a_{22} & a_{23} & a_{24} \\
a_{31} & a_{32} & a_{33} & a_{34} \\
0 & 0 & 0 & 1
\end{array}\right|
$$

where: $n, s, a$ are direction vectors; $P$ is location vector; $a_{i j}$ with $i, j=1 \div 3$ is the direction cosine; $a_{14}, a_{24}, a_{34}$ are elements projected onto the global coordinate system Oxyz as P's components, respectively.

Due to the orthogonal feature of the orient vectors, elements in (3) can be transformed to equation (4). This 
equation is the inverse kinematic problem of industrial robot.

$$
\left\{\begin{array} { l } 
{ s _ { x } = a _ { 1 2 } } \\
{ a _ { x } = a _ { 1 3 } } \\
{ a _ { y } = a _ { 2 3 } } \\
{ p _ { x } = a _ { 1 4 } } \\
{ p _ { y } = a _ { 2 4 } } \\
{ p _ { z } = a _ { 3 4 } }
\end{array} \Leftrightarrow \left\{\begin{array}{l}
s_{x}-a_{12}=0 \\
a_{x}-a_{13}=0 \\
a_{y}-a_{23}=0 \\
p_{x}-a_{14}=0 \\
p_{y}-a_{24}=0 \\
p_{z}-a_{34}=0
\end{array}\right.\right.
$$

Squaring two sides of (4) and add each side has:

$$
\begin{aligned}
& \left(s_{x}-a_{12}\right)^{2}+\left(a_{x}-a_{13}\right)^{2}+\left(a_{y}-a_{23}\right)^{2}+ \\
& \left(p_{x}-a_{14}\right)^{2}+\left(p_{y}-a_{24}\right)^{2}+\left(p_{z}-a_{34}\right)^{2}=0
\end{aligned}
$$

It is clear that (5) is always $\geq 0$ so the minimum of (5) is 0 . Denote $T$ as the objective function in the left hand side:

$$
\begin{aligned}
& T=\left(s_{x}-a_{12}\right)^{2}+\left(a_{x}-a_{13}\right)^{2}+\left(a_{y}-a_{23}\right)^{2} \\
& +\left(p_{x}-a_{14}\right)^{2}+\left(p_{y}-a_{24}\right)^{2}+\left(p_{z}-a_{34}\right)^{2}
\end{aligned}
$$

This function form is well known as Rosenbrock-Banana function [45].

The goal of kinematic control is to ensure the accuracy of the position and direction of the robot end effector. Therefore, it is necessary to determine the value of the joints to meet the requirements of minimum deviation in positions and directions of the end effector as well as to meet the constraint conditions in robot structure.

Denote $f=\left(q_{1}, q_{2}, \ldots q_{n}\right)$ is the vector of joint variables.

Space D determines the value range of the joint variables:

$$
\left\{\begin{array}{l}
L_{1} \leq q_{1} \leq U_{1} \\
L_{2} \leq q_{2} \leq U_{2} \\
\vdots \\
L_{n} \leq q_{n} \leq U_{n}
\end{array}\right.
$$

where: $L_{i}$ and $U_{i}$ are constraints of joint variables.

$T=f(q)$ : function describing the deviation of position and direction of the robot end-effector.

The problem of determining the value of the joint variables is written as follows:

$$
\left\{\begin{array}{l}
T=f\left(q_{1}, q_{2}, \ldots q_{n}\right) \rightarrow \text { minimize } \\
L_{i} \leq q_{i} \leq U_{i} \\
q_{i} \in D ; i=1 \div n
\end{array}\right.
$$

This is a mathematical model of the non-linear multi-variable optimization problem.

\section{Solution Method For the Optimization Problem}

The non-linear optimization problem with general constraints is defined as follows:

$$
\left\{\begin{array}{l}
\operatorname{Min} f(x), x \in F \subset S \subset R^{n} \\
h_{i}(x)=0, i=1,2 \ldots p \\
g_{j}(x) \leq 0, j=p+1, \ldots q \\
l_{k} \leq x_{k} \leq u_{k}, k=1,2 \ldots n
\end{array}\right.
$$

While this optimization problem could be solved by several methods as Sequential Quadric Programming method (SQP), GA, GRG, and so on, the function $f(x)$ in this the optimization problem is the Rosenbrock-Banana function which has complex geometric representation. It is consequently best solved by GRG method [46], [47].

The GRG method is one of the techniques that are based on extending optimization methods for linear constraints applying to nonlinear constraints. This procedure is based on the idea of elimination of variables using the equality constraints. The goal of GRG is converting the constrained problem into an unconstrained one by using direct substitution. The development of the GRG method is followed by that of constrained variation. The approach used in GRG method is both determining an improved direction for the technical model and satisfying the constraint equations [48]. An example of Generalized Reduced Gradient (GRG2) algorithm for optimizing nonlinear problems could be found in Microsoft Excel Solver. This algorithm was developed by Leon Lasdon in University of Texas, and Allan Waren, of Cleveland State University (Microsoft Inc. 2011).

\section{DETERMinAtion OF THE TOLERANCE OF JOINT ANGLE MOVEMENT}

For quantitative assessment of joint manufacturing tolerance, this variable must be described in the form of mathematical model. The joint tolerance is resulted from the allocation of position and direction errors of the end-effector to the build-up joints in the procedure of design. Hence, the model of dynamic joint tolerance must describe the relationship among position and direction tolerances of the end-effector the geometric characteristics of the mechanism and the tolerances of build-up joints.

From the robot kinematic equation formulated as:

$$
\begin{aligned}
& f_{i}\left(q_{1}, q_{2}, \ldots, q_{6}\right)=0 \\
& i=1 \div n
\end{aligned}
$$

where $n$ is the generalized coordinate sufficient to determine the position and direction of end-effector.

Define the current given position in the working space of robot as:

$$
p_{i}=\left(x_{i}, y_{i}, z_{i}, \alpha_{i}, \beta_{i}, \gamma_{i}\right)
$$

where:

$\left(x_{i}, y_{i}, z_{i}\right)$ Describes the position of the end-effector;

$\left(\alpha_{i}, \beta_{i}, \gamma_{i}\right)$ Describes the direction of the end-effector;

The value of joint variable in this condition could be found by solving the following set of equations: 


$$
\begin{aligned}
& f_{i}\left(q_{1}, q_{2}, \ldots, q_{6}\right)=p_{i} \\
& i=1 \div n
\end{aligned}
$$

Assuming that $p_{i}$ in joint space at the given time is demonstrated by:

$$
p_{i}=\left(q_{1}, q_{2}, \ldots, q_{6}\right)^{(i)}
$$

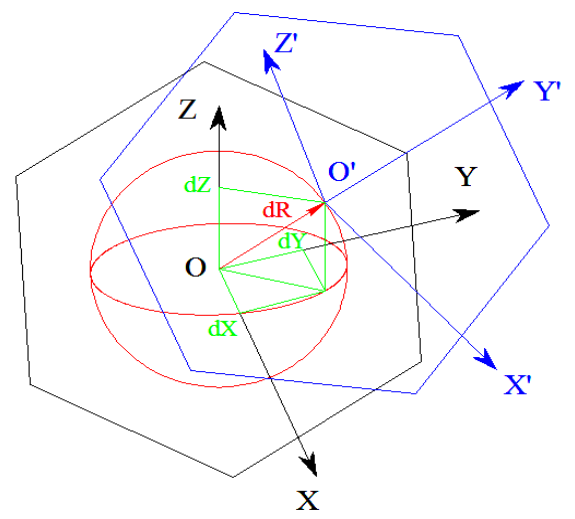

Fig. 2. The movement with the smallest step of moving platform between two points in space.

Considering a hexapod parallel robot in space with moving platform as shown in Fig. 2. Denoting $d R$ as the smallest movement which is the design requirement of accurate performance. These moving quantities can be generalized as a sphere with the radial of $\mathrm{dR}$ and the center at $\mathrm{O}$ - the given point. The design requirement of this problem is that the joint tolerances will be determined from this limited deviation for the identification of free radial movement $\delta$ of the built-up joints.

The tolerances of the built-up joints include radial clearance $\delta$ and axial clearance $\delta_{1}+\delta_{2}$ (Fig. 3).

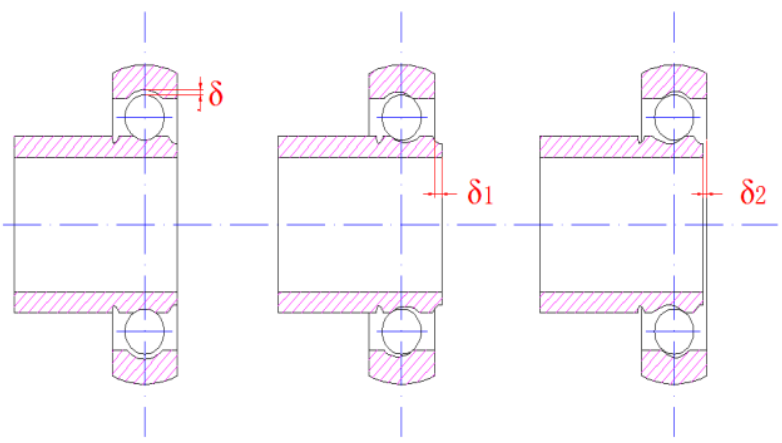

Fig. 3. Several types of clearance to be controlled in joints.

Choosing the smallest resolution axis among three axes $x$, $y, z$ of the robot $O x y z$ coordinate for determining the smallest movement of moving platform from an arbitrary direction to $\mathrm{dR}$ sphere, then identifying the coordinates of the next point where the platform will move to as follows:

$$
p_{i+1}=\left(x_{i+1}, y_{i+1}, z_{i+1}, \alpha_{i+1}, \beta_{i+1}, \gamma_{i+1}\right)
$$

or: $p_{i+1}=\left|\begin{array}{l}p_{i x}+d x \\ p_{i y}+d y \\ p_{i z}+d z\end{array}\right|$

This coordinate will get the correspondent value of joint variables when updating into equation (12) as:

$$
p_{i+1}=\left(q_{1}, q_{2}, \ldots, q_{6}\right)^{(i+1)}
$$

It means that every joint has performed a movement as:

$$
\left\{\begin{array}{l}
\delta q_{1}=q_{1}{ }^{(i+1)}-q_{1}{ }^{(i)} \\
\vdots \\
\delta q_{6}=q_{6}{ }^{(i+1)}-q_{6}{ }^{(i)}
\end{array}\right.
$$

They are the maximum movement of free angle in the range of manufacture deviation limitation. In this case, the calculation results will be converted to the accuracy of transmission system under the mechanism point of view when considering the design of Fig. 4.

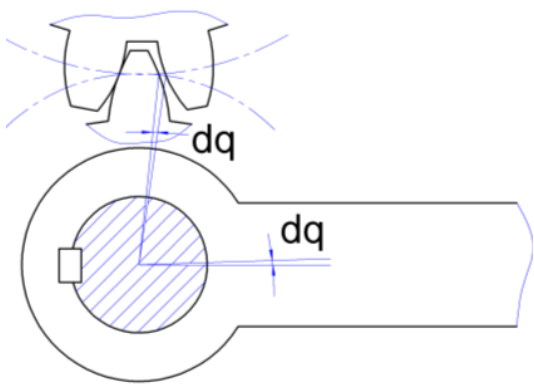

Fig. 4. The transmission deviation of restricted angle caused by mechanic clearance defined by (16)

\section{DETERMINATION OF THE DEVIATION OF LINK DIMENSIONS AND JOINT FREE RADIAL MOVEMENT BY USING INVERSE KINEMATIC}

In Section $\mathrm{V}$, a technique determining the free angle of the joints by using the inverse kinematic problem rather than Jacobian matrix method has been introduced. This method however has a drawback that the radial movements of tolerances in built-up links are unrevealed, so movement of the end-effector in a tolerance limit is unreliable. Besides, the small translation movements appear in Jacobian matrix when it has translation joints in robot.

The full kinematic model of the robot includes the length of built-up links in model (12) as follows:

$$
\begin{aligned}
& f_{i}\left(q_{1}, q_{2}, \ldots, q_{6},\left(l_{i}+\delta l_{i}\right)\right)=0 \\
& i=1 \div n
\end{aligned}
$$

where $l_{i}$ is the nominal length of $i^{\text {th }}$ link; $\delta l_{i}$ is an unknown value called the allowable length deviation of the $i^{\text {th }}$ link. The procedure of determining this value is:

Denote the given point in working space of robot as:

$$
p_{i}=\left(x_{i}, y_{i}, z_{i}, \alpha_{i}, \beta_{i}, \gamma_{i}\right)
$$

where:

$\left(x_{i}, y_{i}, z_{i}\right)$ Describes the position of the end-effector;

$\left(\alpha_{i}, \beta_{i}, \gamma_{i}\right)$ Describes the direction of the end-effector

The value of joint variable in this state can be found from the following equation: 


$$
\begin{aligned}
& f_{i}\left(q_{1}, q_{2}, \ldots, q_{6}\right)=p_{i} \\
& i=1 \div n
\end{aligned}
$$

Assuming that the point $p_{i}$ in joint space at given time can be described by:

$$
p_{i}=\left(q_{1}, q_{2}, \ldots, q_{6}\right)^{(i)}
$$

Combining (20) with the given nominal dimension value of each links $l_{i}$, equation (17) including $n$ variables with the predetermined values $\delta l_{i}$ when establishing the equation of small movements along the free movement of end-effector can be solved by GRG algorithm [46], [47].

When allocate the tolerance of end-effector to the built-up links inversely, the different ranges of calculated tolerance values of each joint are founded at different control points. The choice of tolerance range as in Fig. 5.

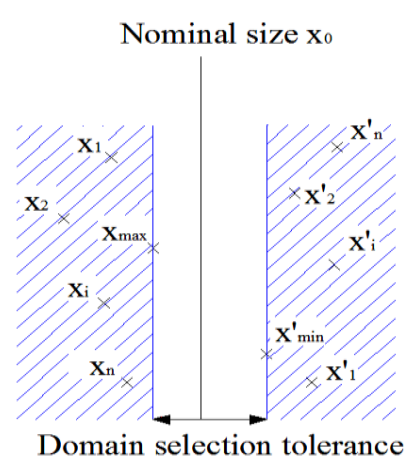

Fig. 5. Tolerance choice of built-up links.

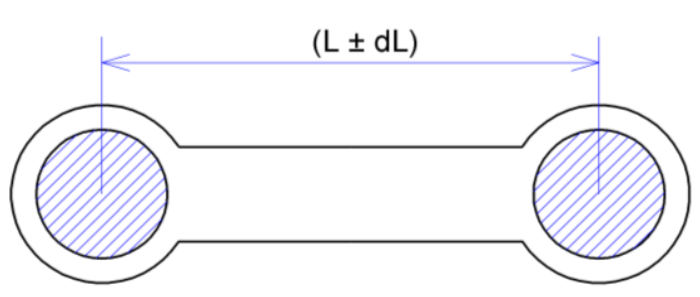

(a). Tolerance of link dimension.

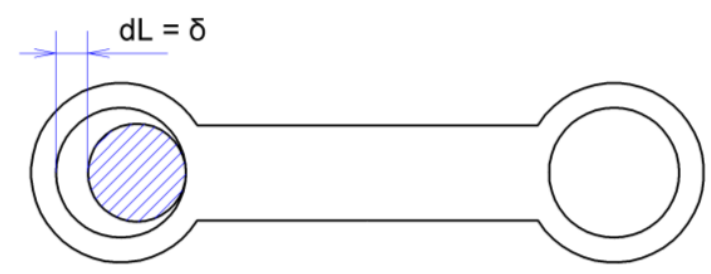

(b). Tolerance of joint clearance

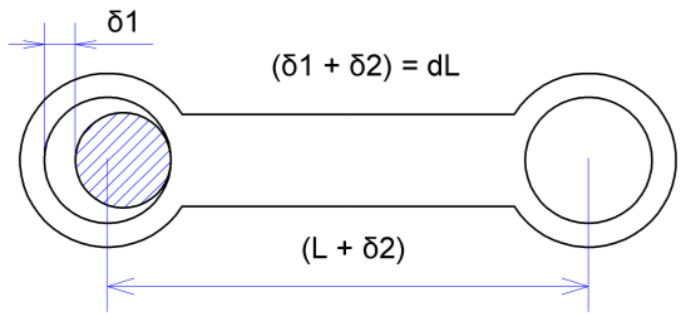

(c). Tolerance allocated into link dimensions and joint clearance Fig. 6. The use of calculated tolerance results.

Considering an arbitrary $i^{\text {th }}$ link at the points from $P_{1}$ to $P_{n}$ in control trajectory, the tolerance range of $i^{\text {th }}$ link changing around the value of nominal dimension can be calculated based on the small movement of the end-effector in inverse kinematic problem. Defining set of the calculated values of link dimensions which are smaller than nominal dimensions is $\left(x_{1}, x_{2}, \ldots x_{n}\right)$. Defining set of the calculated values of link dimensions which are larger than nominal dimensions is $\left(x_{1}^{\prime}, x_{2}^{\prime}, \ldots x_{n}^{\prime}\right)$. For making the tolerance of the end-effector in the range of allowable values, the tolerance of $i^{\text {th }}$ link should be chosen as: the lower limitation equals to max of $\left(x_{1}, x_{2}, \ldots x_{n}\right)$, and the upper tolerance equals to min of $\left(x_{1}^{\prime}, x_{2}^{\prime}, \ldots x_{n}^{\prime}\right)$.

From the calculated tolerance values of built-up links and the design requirements, the manufacture tolerances of both built-up links and joints will be determined as in Fig. 6 .

\section{THE EXAMPLE OF NUMERICAL SiMULATION}

\section{A. Robot Arm}

Considering the kinematic diagram of robot Fanuc S900W in the form of equivalent open-series (Fig. 7), with DH table described in Table I.

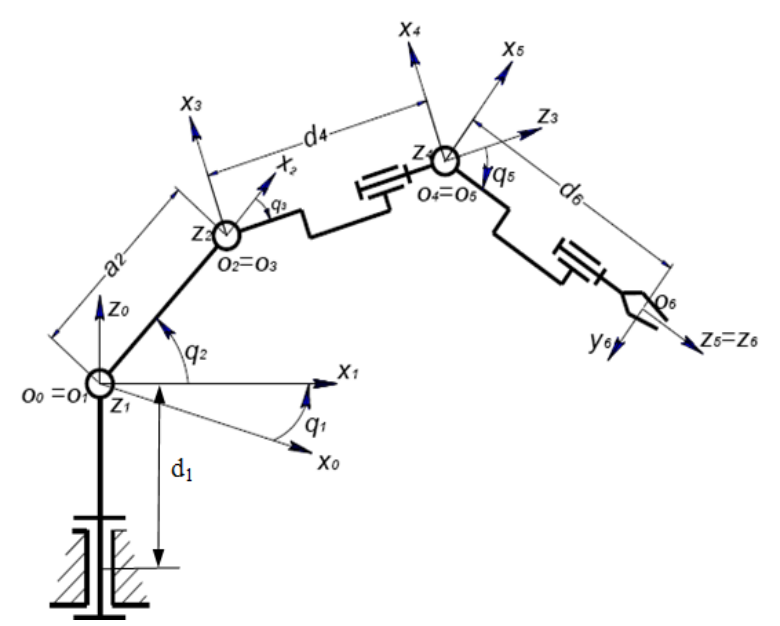

Fig. 7. The equivalent kinematic diagram of robot Fanuc S900W.

TABLE I: KINEMATIC PARAMETERS OF ROBOT FANUC S900W
\begin{tabular}{|c|c|c|c|c|}
\hline Joints & $\boldsymbol{R}(\boldsymbol{z}, \alpha)$ & $\boldsymbol{T}(\boldsymbol{z}, \boldsymbol{d})$ & $\boldsymbol{T}(\boldsymbol{x}, \boldsymbol{a})$ & $\boldsymbol{R}(\boldsymbol{x}, \boldsymbol{\beta})$ \\
\hline 1 & $\left(\alpha_{1}\right)$ & 650 & 0 & 90 \\
\hline 2 & $\left(\alpha_{2}\right)$ & 0 & 700 & 0 \\
\hline 3 & $\left(\alpha_{3}\right)$ & 0 & 0 & 90 \\
\hline 4 & $\left(\alpha_{4}\right)$ & 675 & 0 & -90 \\
\hline 5 & $\left(\alpha_{5}\right)$ & 0 & 0 & 90 \\
\hline 6 & $\left(\alpha_{6}\right)$ & 300 & 0 & 0 \\
\hline
\end{tabular}

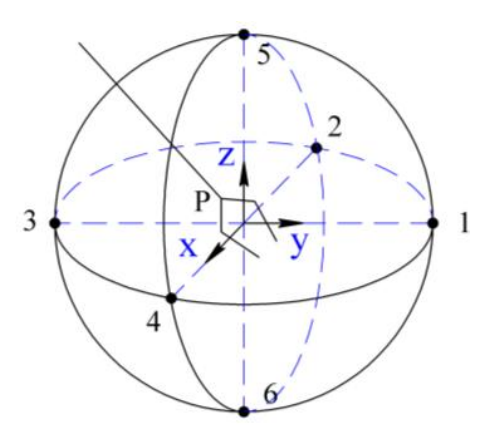

Fig. 8. Six allowable moving points of the end-effector in the limited deviation range of a sphere. 
Requirement: Determine the manufacture tolerances with the dimensions of $650,700,675,300$ in $\mathrm{DH}$ table, the given condition of uncontrolled deviation of arm center, the end-effector, is no higher than $0.2 \mathrm{~mm}$ in all directions in working space ( $\mathrm{dR}=0.2 \mathrm{~mm}$, Fig. 8$)$.

In Fig. 8 are the six allowable moving points of the end-effector in the limited deviation range of a sphere. The coordinates of these points can be calculated from the sphere center (the desired nominal position of robot end-effector center) and the given diameter of the sphere (the limited tolerance of the end-effector).

Considering the study points in the working space of robot, the inverse kinematic problem and applying the equations from (14) to (19), the study tolerances of the built-up links of robot Fanuc S900W are presented in Table II.

Applying the choice of tolerance range as in Fig. 5, the tolerance range of each link is shown in Table III.

TABLE II: EXTRACTED RESULTS OF MEASURED TOLERANCES OF BUILT-UP LINKS IN ROBOT FANUC S900W

\begin{tabular}{|c|c|c|c|c|c|c|c|}
\hline Points & $P x$ & $P y$ & $P z$ & $d 1(\mathrm{~mm})$ & $a 2(\mathrm{~mm})$ & $d 3+d 4$ & $d 5+d 6$ \\
\hline$P 1$ & -127.88 & -302 & 408 & 650 & 700 & 675 & 300 \\
\hline 1 & -127.88 & -301.8 & 408 & 650.2324 & 699.8633 & 674.6555 & 299.6622 \\
\hline 2 & -128.08 & -302 & 408 & 650.1342 & 699.8544 & 674.9514 & 299.6714 \\
\hline 3 & -127.88 & -302.2 & 408 & 649.7255 & 700.088 & 675.3291 & 300.3378 \\
\hline 4 & -127.68 & -302 & 408 & 649.8237 & 700.0969 & 675.0331 & 300.3286 \\
\hline 5 & -127.88 & -302 & 408.2 & 650.0981 & 699.8821 & 674.9626 & 300 \\
\hline 6 & -127.88 & -302 & 407.8 & 649.8598 & 700.0692 & 675.0219 & 300 \\
\hline$P 2$ & -120 & -288 & 410 & 650 & 700 & 675 & 300 \\
\hline 1 & -120 & -287.8 & 410 & 650.3098 & 699.833 & 674.6188 & 299.5684 \\
\hline 2 & -120.2 & -288 & 410 & 650.2908 & 699.7667 & 674.844 & 299.464 \\
\hline 3 & -120 & -288.2 & 410 & 649.6515 & 700.1217 & 675.3646 & 300.4316 \\
\hline 4 & -119.8 & -288 & 410 & 649.6705 & 700.1879 & 675.1394 & 300.5358 \\
\hline 5 & -120 & -288 & 410.2 & 650.1024 & 699.8857 & 674.9581 & 300 \\
\hline 6 & -120 & -288 & 409.8 & 649.8589 & 700.069 & 675.0253 & 300 \\
\hline$P 3$ & 200 & 300 & 250 & 650 & 700 & 675 & 300 \\
\hline 1 & 200 & 300.2 & 250 & 650.0537 & 700.1361 & 674.8457 & 299.8346 \\
\hline 2 & 199.8 & 300 & 250 & 650.2936 & 699.9129 & 674.7622 & 299.4519 \\
\hline 3 & 200 & 299.8 & 250 & 649.9283 & 699.8518 & 675.1346 & 300.1654 \\
\hline 4 & 200.2 & 300 & 250 & 649.6883 & 700.075 & 675.2181 & 300.5481 \\
\hline 5 & 200 & 300 & 250.2 & 650.115 & 699.9434 & 674.9073 & 300 \\
\hline 6 & 200 & 300 & 249.8 & 649.8669 & 700.0446 & 675.073 & 300 \\
\hline$P 4$ & 450 & 400 & 450 & 650 & 700 & 675 & 300 \\
\hline 1 & 450 & 400.2 & 450 & 650.2455 & 700.2147 & 674.6973 & 299.3779 \\
\hline 2 & 449.8 & 400 & 450 & 650.3459 & 699.9291 & 674.6647 & 299.3137 \\
\hline 3 & 450 & 399.8 & 450 & 649.7254 & 699.7787 & 675.2713 & 300.6221 \\
\hline 4 & 450.2 & 400 & 450 & 649.625 & 700.0642 & 675.3039 & 300.6863 \\
\hline 5 & 450 & 400 & 450.2 & 650.0953 & 699.9761 & 674.8869 & 300 \\
\hline 6 & 450 & 400 & 449.8 & 649.8757 & 700.0173 & 675.0817 & 300 \\
\hline$P 5$ & 299 & 302 & 300 & 650 & 700 & 675 & 300 \\
\hline 1 & 299 & 302.2 & 300 & 650.0724 & 700.1453 & 674.849 & 299.7822 \\
\hline 2 & 298.8 & 302 & 300 & 650.2267 & 699.9132 & 674.8369 & 299.5793 \\
\hline 3 & 299 & 301.8 & 300 & 649.919 & 699.8496 & 675.1413 & 300.2178 \\
\hline 4 & 299.2 & 302 & 300 & 649.7648 & 700.0816 & 675.1534 & 300.4207 \\
\hline 5 & 299 & 302 & 300.2 & 650.1205 & 699.9519 & 674.9096 & 300 \\
\hline 6 & 299 & 302 & 299.8 & 649.8709 & 700.0429 & 675.0806 & 300 \\
\hline
\end{tabular}

TABLE III: THE RESULTS OF DIMENSION TOLERANCES IN BUILT-UP LINKS OF ROBOT FANUC S900W

\begin{tabular}{|c|c|c|c|c|}
\hline Links & $\boldsymbol{d 1}(\mathbf{m m})$ & $\boldsymbol{a 2}(\mathbf{m m})$ & $\boldsymbol{d 3}+\boldsymbol{d} \mathbf{4}$ & $\boldsymbol{d 5}+\boldsymbol{d 6}$ \\
\hline $\begin{array}{c}\text { Nominal } \\
\text { dimensions }\end{array}$ & 650 & 700 & 675 & 300 \\
\hline $\begin{array}{c}\text { Upper limit } \\
\text { tolerance }\end{array}$ & 649.9283 & 699.9761 & 674.9626 & 299.8346 \\
\hline $\begin{array}{c}\text { Lower limit } \\
\text { tolerance }\end{array}$ & 650.0537 & 700.0173 & 675.0219 & 300.1654 \\
\hline Tolerance range & 0.1254 & 0.0412 & 0.0593 & 0.3308 \\
\hline
\end{tabular}

Depending on the design requirement, these results can be used as the basis of joint clearance choice (Fig. 3) or allocation tolerances of link dimensions as illustrated in Fig.
6.

\section{B. Parallel Robot}

Considering the tolerance calculation example of built-up links in 3RRR parallel robot with the deviation of the end-effector $\mathrm{dR} \leq 0.01(\mathrm{~mm})$ in all directions in Oxy coordinate (Fig. 9).

The structural nominal parameters of robot are: moving platform and base platform are equiangular triangles with side edges as $h=300(\mathrm{~mm}), c=600(\mathrm{~mm})$ respectively and the nominal dimensions of links $a_{i}=400(\mathrm{~mm}), b_{i}=300(\mathrm{~mm})$. The problem is determining the manufacturing tolerances of $h, c, a_{i}, b_{i}$ dimensions with the tolerance of the end-effector 
$\leq 0.01(\mathrm{~mm})$.

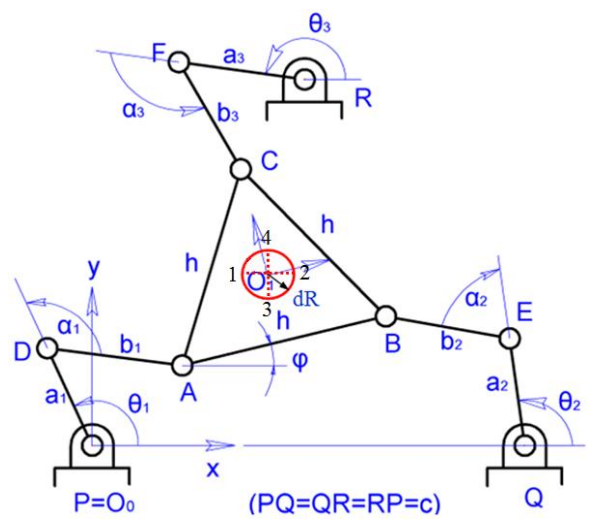

Fig. 9. The 3-RRR planar parallel robot.

In Fig. 9, the points 1, 2, 3, 4 describe four allowable maximum deviation points of end-effector in the deviation cycle with center $\mathrm{O}_{1}$ and radius $\mathrm{dR}=0.01 \mathrm{~mm}$.

The center $\mathrm{O}_{1}$, the desired nominal position of moving platform, and the given radius, $\mathrm{dR}=0.01 \mathrm{~mm}$, can be determined from coordinates of these points.

Considering the study points in the working space of robot, the inverse kinematic problem and applying the equations from (14) to (19), the study tolerances of the built-up links of 3RRR robot are presented in Table IV.

TABLE IV: EXTRACTED RESULTS OF MEASURED TOLERANCES IN BUILT-UP LINKS OF 3RRR PARALLEL ROBOT

\begin{tabular}{|c|c|c|c|c|c|c|}
\hline Points & $\boldsymbol{P} \boldsymbol{x}(\mathbf{m m})$ & $\boldsymbol{P} \boldsymbol{(}(\mathbf{m m})$ & $\boldsymbol{a}(\mathbf{m m})$ & $\boldsymbol{b}(\mathbf{m m})$ & $\boldsymbol{c}(\mathbf{m m})$ & $\boldsymbol{h}(\mathbf{m m})$ \\
\hline$P 1$ & 181.205 & 403.802 & 400 & 300 & 600 & 300 \\
\hline 1 & 181.195 & 403.802 & 400.0077 & 300.0057 & 599.9845 & 299.9787 \\
\hline 2 & 181.215 & 403.802 & 399.9922 & 299.9942 & 600.0154 & 300.0212 \\
\hline 3 & 181.205 & 403.792 & 399.9866 & 299.9899 & 599.992 & 300.0020 \\
\hline 4 & 181.205 & 403.812 & 400.0133 & 300.0100 & 600.0079 & 299.9979 \\
\hline$P 2$ & 213.081 & 419.724 & 400 & 300 & 600 & 300 \\
\hline 1 & 213.071 & 419.724 & 400.0077 & 300.0058 & 599.9833 & 299.9775 \\
\hline 2 & 213.091 & 419.724 & 399.9922 & 299.9941 & 600.0166 & 300.0224 \\
\hline 3 & 213.081 & 419.714 & 399.9865 & 299.9898 & 599.9941 & 300.0042 \\
\hline 4 & 213.081 & 419.734 & 400.0134 & 300.0101 & 600.0058 & 299.9957 \\
\hline$P 3$ & 245.611 & 434.263 & 400 & 300 & 600 & 300 \\
\hline 1 & 245.601 & 434.263 & 400.0078 & 300.0059 & 599.9821 & 299.9762 \\
\hline 2 & 245.621 & 434.263 & 399.9921 & 299.9940 & 600.0178 & 300.0237 \\
\hline 3 & 245.611 & 434.253 & 399.9863 & 299.9897 & 599.9962 & 300.0065 \\
\hline 4 & 245.611 & 434.273 & 400.0136 & 300.0102 & 600.0037 & 299.9934 \\
\hline$P 4$ & 278.794 & 447.237 & 400 & 300 & 600 & 300 \\
\hline 1 & 278.784 & 447.237 & 400.0080 & 300.0060 & 599.9808 & 299.9748 \\
\hline 2 & 278.804 & 447.237 & 399.9919 & 299.9939 & 600.0191 & 300.0251 \\
\hline 3 & 278.794 & 447.227 & 399.9860 & 299.9895 & 599.9985 & 300.0089 \\
\hline 4 & 278.794 & 447.247 & 400.0139 & 300.0104 & 600.0014 & 299.991 \\
\hline$P 5$ & 312.647 & 458.344 & 400 & 300 & 600 & 300 \\
\hline 1 & 312.637 & 458.344 & 400.0083 & 300.0062 & 599.9794 & 299.9732 \\
\hline 2 & 312.657 & 458.344 & 399.9916 & 299.9937 & 600.0205 & 300.0267 \\
\hline 3 & 312.647 & 458.334 & 399.9856 & 299.9892 & 600.0009 & 300.0117 \\
\hline 4 & 312.647 & 458.354 & 400.0143 & 300.0107 & 599.999 & 299.9882 \\
\hline$P 6$ & 347.188 & 467.062 & 400 & 300 & 600 & 300 \\
\hline 1 & 347.178 & 467.062 & 400.0086 & 300.0064 & 599.9779 & 299.9714 \\
\hline 2 & 347.198 & 467.062 & 399.9913 & 299.9935 & 600.022 & 300.0285 \\
\hline 3 & 347.188 & 467.052 & 399.9849 & 299.9887 & 600.0035 & 300.0147 \\
\hline 4 & 347.188 & 467.072 & 400.01500 & 300.0112 & 599.9964 & 299.9852 \\
\hline$P 7$ & 382.398 & 472.401 & 400 & 300 & 600 & 300 \\
\hline 1 & 382.388 & 472.401 & 400.0091 & 300.0068 & 599.9762 & 299.9693 \\
\hline 2 & 382.408 & 472.401 & 399.9908 & 299.9931 & 600.0237 & 300.0306 \\
\hline 3 & 382.398 & 472.391 & 399.9841 & 299.9880 & 600.0065 & 300.0184 \\
\hline 4 & 382.398 & 472.411 & 400.0158 & 300.0119 & 599.9934 & 299.9815 \\
\hline & & & & & & \\
\hline
\end{tabular}

\begin{tabular}{|c|c|c|c|c|c|c|}
\hline$P 8$ & 417.965 & 472.138 & 400 & 300 & 600 & 300 \\
\hline 1 & 417.955 & 472.138 & 400.0100 & 300.0075 & 599.974 & 299.9665 \\
\hline 2 & 417.975 & 472.138 & 399.9899 & 299.9924 & 600.0259 & 300.0334 \\
\hline 3 & 417.965 & 472.128 & 399.9826 & 299.9870 & 600.0102 & 300.0232 \\
\hline 4 & 417.965 & 472.148 & 400.0173 & 300.0129 & 599.9897 & 299.9767 \\
\hline$P 9$ & 451.03 & 460.044 & 400 & 300 & 600 & 300 \\
\hline 1 & 451.02 & 460.044 & 400.0115 & 300.0086 & 599.9712 & 299.9625 \\
\hline 2 & 451.04 & 460.044 & 399.9884 & 299.9913 & 600.0287 & 300.0374 \\
\hline 3 & 451.03 & 460.034 & 399.9799 & 299.9849 & 600.0151 & 300.0301 \\
\hline 4 & 451.03 & 460.054 & 400.0200 & 300.0150 & 599.9848 & 299.9698 \\
\hline$P 10$ & 464.521 & 428.604 & 400 & 300 & 600 & 300 \\
\hline 1 & 464.511 & 428.604 & 400.0143 & 300.0107 & 599.9681 & 299.9573 \\
\hline 2 & 464.531 & 428.604 & 399.9856 & 299.9892 & 600.0318 & 300.0426 \\
\hline 3 & 464.521 & 428.594 & 399.9750 & 299.9812 & 600.0205 & 300.0392 \\
\hline 4 & 464.521 & 428.614 & 400.0249 & 300.0187 & 599.9794 & 299.9607 \\
\hline$P 11$ & 454.378 & 394.708 & 400 & 300 & 600 & 300 \\
\hline 1 & 454.368 & 394.708 & 400.0174 & 300.0130 & 599.9665 & 299.9534 \\
\hline 2 & 454.388 & 394.708 & 399.9825 & 299.9869 & 600.0334 & 300.0465 \\
\hline 3 & 454.378 & 394.698 & 399.9697 & 299.9773 & 600.0233 & 300.0459 \\
\hline 4 & 454.378 & 394.718 & 400.0302 & 300.0226 & 599.9766 & 299.9540 \\
\hline$P 12$ & 435.335 & 364.645 & 400 & 300 & 600 & 300 \\
\hline 1 & 435.325 & 364.645 & 400.0203 & 300.0152 & 599.9662 & 299.9509 \\
\hline 2 & 435.345 & 364.645 & 399.9796 & 299.9847 & 600.0337 & 300.0490 \\
\hline 3 & 435.335 & 364.635 & 399.9646 & 299.9735 & 600.0238 & 300.0503 \\
\hline 4 & 435.335 & 364.655 & 400.0353 & 300.0264 & 599.9761 & 299.9496 \\
\hline & & & & & & \\
\hline
\end{tabular}

Similarly, by applying the method of tolerance range determination as shown in Fig. 5, the tolerance range of the built-up links is presented in Table $\mathrm{V}$.

TABLE V: The TOLERANCE RESUltS OF BUILT-UP LINK DiMENTIONS OF 3RRR PARALLEL ROBOT

\begin{tabular}{|l|c|c|c|c|}
\hline Links & $\boldsymbol{a}(\mathbf{m m})$ & $\boldsymbol{b}(\mathbf{m m})$ & $\boldsymbol{c}(\mathbf{m m})$ & $\boldsymbol{h}(\mathbf{m m})$ \\
\hline $\begin{array}{c}\text { Nominal } \\
\text { dimensions }\end{array}$ & 400 & 300 & 600 & 300 \\
\hline $\begin{array}{c}\text { Upper limit } \\
\text { tolerance }\end{array}$ & 400.0077 & 300.0058 & 600.0009 & 300.0117 \\
\hline $\begin{array}{c}\text { Lower limit } \\
\text { tolerance }\end{array}$ & 399.9922 & 299.9942 & 599.9991 & 299.9883 \\
\hline $\begin{array}{c}\text { Tolerance } \\
\text { range }\end{array}$ & 0.01543 & 0.01157 & 0.00182 & 0.02341 \\
\hline
\end{tabular}

Depending on the design requirement, these results can be used as the basis of joint clearance choice (Fig. 3) or allocation tolerances of link dimensions as illustrated in Fig. 6.

\section{Checking the Accuracy of the Proposed Method}

In robot design technique, the manufacturing tolerances of the build-up joints are calculated to meet the separated design requirements as accuracy, manufacture cost and so on. The measured tolerances must meet the requirement of the initial design.

Because the values of tolerances are small in nature, and the robot kinematic problem includes variety of transcendental functions which require round-off their numbers, the numerical method is an approximate approach. Hence,

$$
\text { Required tolerance }=\text { round-off error }+ \text { method error. }
$$

The proposed method in this paper, however utilize the highly accurate GRG, the calculated results of tolerance are high accuracy and reliability. As a demonstration, an example is given as follows.

Taking the tolerance result of 3RRR parallel robot in which 
the end-effector error $\mathrm{dR} \leq 0.01 \mathrm{~mm}$ in all dimensions in working space.

Considering a trajectory across 12 key points belong to an ellipse in robot working space (Fig. 10). The tolerances result in Table $\mathrm{V}$. The errors of the moving platform when following this trajectory are shown in Fig. 11.

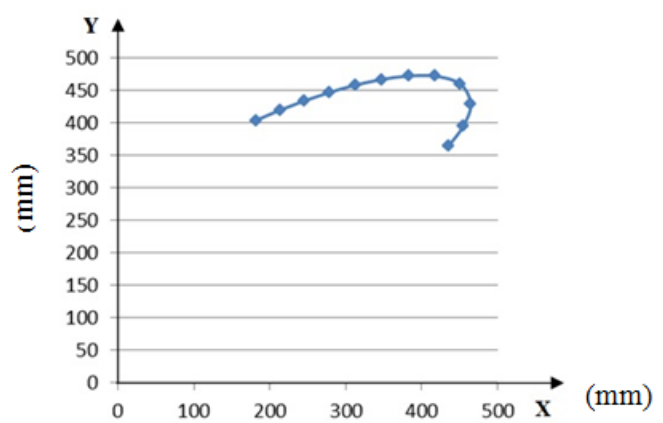

Fig. 10. The moving trajectory across twelve points in an ellipse.

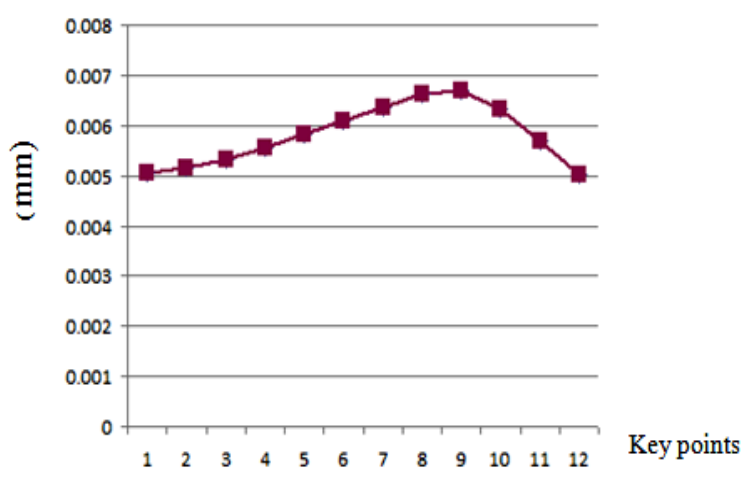

Fig. 11. The errors of control trajectory.

It can be seen that the errors of all the measured points on control trajectory are in the range of $0.005 \mathrm{~mm}$ to $0.0067 \mathrm{~mm}$ (smaller than $0.01 \mathrm{~mm}$ ). It means that the calculated tolerances of the component links satisfy the requirement on the accuracy of the initial design.

\section{CONCLUSION}

The proposed method both considers the effect of link and joint tolerances to the drive system simultaneously and give the instruction of determining the tolerance of each link in the kinematic chain based on the nature of kinematic reaction of the movement accompanied with mathematical statistics. This method overcomes the drawback mathematic models as the squared requirement of inversed matrix in Jacobian matrix. In particularly, with the different mathematic models in different stage of mechatronics designation, the proposed method warrants the solidity of the object designed by applying a unique mathematic model to both kinematic and manufactures tolerance problems.

The solution of spatial dimensions with several constraints to allocate the tolerance of the end-effector into the tolerances of the component links is neglected. The results are calculated in both direct and reverse directions and experiment on mathematic model easily. Besides the widen application on different type of robot as serial robot, parallel robot which has either redundant or under actuated drive, the proposed method is more fitted with the nature of mechanism than the conventional method of establishing and solving the dimensional chain.

\section{REFERENCES}

[1] B. K. Rout and R. K. Mittal, "Tolerance design of manipulator parameters using design of experiment approach," Struct Multidisc Optim, vol. 34, pp. 445-462, 2007

[2] J. W. Wittwer, K. W. Chase, and L. L. Howell, "The direct linearization method applied to position error in kinematic linkages," Mech. Machine Theory, vol. 39, pp. 681-693, 2004

[3] W. D. Wu and S. S. Rao, "Uncertainty analysis and allocation of joint tolerances in robot manipulators based on interval analysis," Reliability Engineering and System Safety, vol. 92, pp. 54-64, 2007.

[4] B. K. Rout and R. K. Mittal, "Optimal manipulator parameter tolerance selection using evolutionary optimization technique," Eng. Appl. Artif. Intell, vol. 21, pp. 509-524, 2008.

[5] J. Kim, W.-J. Song, and B.-S. Kang, "Stochastic approach to kinematic reliability of open-loop mechanism with dimensional tolerance," Applied Mathematical Modelling, vol. 34, pp. 1225-1237, 2010.

[6] S. P. Ji, X. L. Li, and R. Du, "Tolerance synthesis using second-order fuzzy comprehensive evaluation and genetic algorithm," INT.J.PROD.RES, vol. 38, no. 15, pp. 3471-3483, 2000.

[7] A. Ballu and L. Matheu, "Analysis of dimensional and geometrical specifications: standards and models," in Proc. 3rd CIRP Seminars on Computer Aided Tolerancing, 1993, pp. 157-170.

[8] W. Michael and J. N. Siddall, "The optimal tolerance assignment with less than full acceptance," ASME J Mech Des, vol. 104, pp. 855-860, 1982.

[9] D. B. Parkinson, "The application of a robust design method to tolerancing," Trans ASME J Mech Des, vol. 122, pp. 149-154, 2000.

[10] R. S. Rao, A. Asaithambi, and S. K. Agrawal, "Inverse kinematic solution of robot manipulators using interval analysis," ASME J Mech Des, vol. 120, no. 1, pp. 147-150, March 1, 1998.

[11] M. H. Gadallah and H. A. ElMaraghy, "A new algorithm for discrete tolerance optimization," in Proc. Fourth International Conference on Computer Integrated Manufacturing and Automation Technology, IEEE Computer Society Press, 1994, pp. 292-297.

[12] H.-G. R. Choi, M.-H. Park, and E. Salisbury, "Optimal tolerance allocation with loss functions," J Manuf Sci Eng Trans ASME, vol. 122, pp. 529-535, 2000.

[13] B.-R. Cho, Y. J. Kim, D. L. Kimbler, and M. D. Phillips, "An integrated joint optimization procedure for robust and tolerance design," Int J Prod Res, vol 38, pp. 2309-2325, 2000.

[14] C.-X. Feng, J. Wang, and J.-S. Wang, "An optimization model for concurrent selection of tolerances and suppliers," Comput Indust Eng , vol. 40, no. 1-2, pp. 15-33, June 2001.

[15] B. K. Rout and R. K. Mittal, "A review on minimizing variability in product performance with the help of computational tools by controlling active and noise factor for robust design," in Proc. the National Conference on Current Applications of Computers in Design Engineering, Feb. 2001, pp. 13-23.

[16] A. N. Haq, K. Sivakumar, R. Saravanan, and V. Muthiah, "Tolerance design optimization of machine elements using genetic algorithm," Int J Adv Manuf Technol, vol. 25, 2005.

[17] J. J. Paredis and P. K. Khosla, "Agent-based design of fault tolerant manipulators for satellite docking," in Proc. the IEEE International Conference on Robotics and Automation, 1997, vol. 4, pp. 3473-3480

[18] A. H. Aguirre et al., "Using a new GA-based multi-objective optimization technique for the design of robot arms," Robotica, vol. 16, pp. 401-414, 1998.

[19] G. H. Zhuan, J. Wu, and W. Huang, "Optimal planning of robot calibration experiments by genetic algorithms," Journal of Robotic Systems, vol. 14, pp. 741-752, 1997.

[20] S. Ji, X. Li, Y. Ma, and H. Cai, "Optimal tolerance allocation based on fuzzy comprehensive evaluation and genetic algorithm," Int $J$ Adv Manuf Technol, vol. 16, no. 7, pp. 461-468, 2000.

[21] S. Ji, X. Li, and R. Du, "Tolerance synthesis using second- order fuzzy comprehensive evaluation and genetic algorithm," Int J Prod Res, vol. 38 , no. 15 , pp. 3471-3483, 2000.

[22] T.-C. Chen and G. W. Fischer, "A GA-based search method for the tolerance allocation problem," Int J Artif Intell Eng, vol. 14, pp. 133-141, 2000.

[23] D. Zhang, Z. Xu, C. M. Mechefske, and F. Xi, "Optimum design of parallel kinematic tool-heads with genetic algorithms," Robotica, vol. 22, pp. 77-84, 2004 
[24] P. T. Zacharia and N. A. Aspragathos, "Optimal robot task scheduling based on genetic algorithms," Robotics and Computer-Integrated Manufacturing, vol. 21, pp. 67-79, 2005.

[25] P. Kopardekar and S. Anand, "Tolerance allocation using neural networks," Int J Adv Manuf Technol, vol. 10, pp. 269-276, 1995.

[26] E. Dupinet, M. Balazinski, and E. Czogala, "Tolerance allocation based on fuzzy logic and simulated annealing," J Intell Manuf, vol. 7, pp. 487-497, 1996.

[27] J. J. Paredis and P. K. Khosla, "Kinematic design of serial link manipulators from task specifications," International Journal of Robotics Research, vol. 12, no. 3, pp. 274-287, 1993.

[28] H. Zhuang and W. Huang, "Optimal selection of measurement configurations for robot calibration using simulated annealing," in Proc. IEEE Int. Conf. Robotics and Automation, 1994, pp. 393-398.

[29] P. K. Bhatti and S. S. Rao, "Reliability analysis of robot manipulator," Trans. ASME, J. Mech. Transm. Automat. Design., vol. 110, pp. 175-181, 1998.

[30] S. S. Rao and P. K. Bhatti, "Probabilistic approach to manipulator kinematics and dynamics," Reliab. Eng. Syst. Safety, vol. 72, pp. 47-58, 2001

[31] W. J. Lee and T. C. Woo, "Tolerances: their analysis and synthesis," $J$. Eng Ind, vol. 112/113, pp. 113-121, 1990.

[32] J. Zhu and K. L. Ting, "Uncertainty analysis of planar and spatial robots with joint clearances," Mech. Mach. Theory, vol. 35, pp. 1239-1256, 2000.

[33] K. L. Ting, J. Zhu, and D. Watkins, "The effects of joint clearance on position and orientation deviation of linkages and manipulators," Mech. Mach. Theory, vol. 35, pp. 391-401, 2000.

[34] S. S. Rao and P. K. Bhatti, "Probabilistic approach to manipulator kinematics and dynamics," Reliab Eng Syst Saf, vol. 72, pp. 47-58, 2001.

[35] R. Riemer and Y. Edan, "Evaluation of influence of target location on robot repeatability," Robotica, vol. 18, pp. 443-449, 2000.

[36] B. K. Rout and R. K. Mittal, "Parametric tolerance design of manipulator," in Proc. the Conference on Emerging Trends of Mechanical Engineering, March28-29, 2005, pp. 55-61.

[37] B. K. Rout and R. K. Mittal, "Parametric robot manipulator design optimization using Taguchi method," in Proc. the 48th Congress Indian Society for Theoretical and Applied Mechanics, Dec. 2003, pp. 15-27.

[38] B. K. Rout and R. K. Mittal, "Optimal manipulator tolerance design using hybrid evolutionary optimization technique," International Journal of Robotics and Automation, vol. 22, no. 4, 2007.

[39] A. Maciejewski, "Fault tolerant properties of kinematically redundant manipulators," in Proc. IEEE Int. Conf. Robot. Auto, 1990, pp. 638-642.

[40] A. Maciejewski, "The design and control of fault tolerant robots for use in hazardous or remote environments," Robot. Remote Syst.: Proc. Fourth Am. Nucl. Soc., pp. 633-642, 1991.

[41] C. L. Lewis and A. A. Maciejewski, "Dexterity optimization of kinematically redundant manipulators in the presence of joint failures," Comput. Electr. Eng., vol. 20, no. 3, pp. 273-288, 1994.

[42] M. Hassan and L. Notash, "Design modification of parallel manipulators for optimum fault tolerance to joint jam," Mechanism and Machine Theory, vol. 40, pp. 559-577, 2005.

[43] P. B. Khôi, Đ. A. Tuấn, Nguyễn et al., "Khảo sát độ chính xác của robot cấu trúc nối tiếp chuỗi hở," Tuyển tập công trình khoa học Hội nghị Khoa học và Công nghệ toàn quốc về Co khi lần thú III, Việt Nam, 2013.

[44] M. Vukobratovic and B. Borovac, "Accuracy of the robot positioning and orientation assessed via its manufacturing tolerances," Mech. Mach. Theory, vol. 30, no. 1, pp. 11-32, 1995.

[45] L. Yan and D. Ma, "Global optimization for constrained nonlinear programs using line-up competition algorithm," Compus. Oper. Res, vol. 25, pp. 1601-1610, 2001.
[46] L. S. Lasdon, A. D. Warren, A. Jain, and M. Ratner, "Design and Testing of a generalized reduced gradient code for nonlinear programming," ACM Trans. Math. Software, vol. 4, no. 1, pp. 34-50, 1978.

[47] C. Kao, "Performance of several nonlinear programming software packages on microcomputers," Comput. Oper. Res, vol. 25, no. 10, pp 807-816, 1998.

[48] F. Faluyi and C. Arum, "Design optimization of plate girder using generalized reduced gradient and constrained artificial bee colony algorithms," International Journal of Emerging Technology and Advanced Engineering, vol. 2, issue 7, July 2012.

[49] P. Armitage and G. Berry, The Normal Distribution in Statistical Methods in Medical Research, 3rd ed. Backwell Scientific Publication, 1994, pp. 66-71

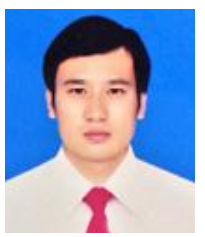

Trang Thanh Trung was born in Gang Thep, Tha Nguyen City, Viet Nam in 1983. He is a Ph.D. candidate at the School of Mechanical and Automotive Engineering, South China University of Technology, China. He received the B.S, M.S. degrees in mechanical engineering from Thai Nguyen University, Viet Nam in 2006, 2010, respectively.

From 2008 to 2012, he was a lecturer at Thai Nguyen University of Technology, Viet Nam. His research interest covers robot control technology and machine learning. There were 2 articles published in magazines and conferences. Corresponding author of this paper.

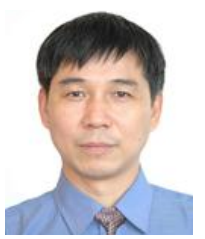

Li Wei Guang was born in 1958 , he is a $\mathrm{PhD}$, professor and doctoral supervisor. He received the B.S, M.S. and $\mathrm{Ph} . \mathrm{D}$. degrees in mechanical engineering from the Mechanical and Automotive Engineering, South China University of Technology, China in 1982, 1996, 1999, respectively.

From 2001 to 2015 , he was a director of the research of modern NC Technology in South China University of Technology; the vice president of Guangdong Manufacturing Information Society, director of Hardware Products Standardization Technical Committee. His research interest covers modern $\mathrm{CNC}$ equipment, manufacturing system of digital and information control, industrial robot technology, electromechanical integration equipment, steam turbine bearing and vibration reduction technology.

He has been more than 10 technical achievements in enterprise application and industrialization of new technologies for CNC Machining Center. He has won more than 30 national invention patent and utility model patents. In recent years, he has more than 100 papers published in national and international academic papers, editors published a modern manufacturing technology (mechanical industry publishing house), mechanical control Foundation (bilingual tutorial) (Wuhan University of technology press), the machinery and equipment of CNC technology (national defense industry press) and 3 textbooks.

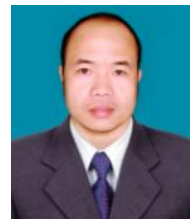

Pham Thanh Long was born in Vinh Phu, Viet Nam in 1977. He received the B.S, M.S. and Ph.D. degrees in mechanical engineering from the Faculty of Mechanica Engineering, Thai Nguyen University of Technology, Viet Nam in 1995, 2001, 2006, respectively.

From 2000 to 2008, he was a lecturer at the Faculty of Mechanical Engineering, Thai Nguyen University of Technology, Viet Nam. From 2008 to 2015, he was the head of Department of Mechatronics, Thai Nguyen University of Technology. His research interest covers mechatronics, robotics, automation in manufacturing, $\mathrm{CAD} / \mathrm{CAM} / \mathrm{CNC}$ technology. He has more than 17 papers published in national and international academic papers, 03 textbooks, and participated in 7 research topics in Vietnam. 